\title{
Magneto-electric control of polarization in spintronic terahertz emitters
}

\author{
Nicolas Tiercelin ${ }^{1}$, Geoffrey Lezier ${ }^{1}$, Pierre Koleják ${ }^{1,2}$, Jean-François Lampin ${ }^{1}$, \\ Yannick Dusch ${ }^{1}$, Kamil Postava ${ }^{2}$ and Mathias Vanwolleghem ${ }^{1}$ \\ ${ }^{1}$ Univ. Lille, CNRS, Centrale Lille, Univ. Polytechnique Hauts-de-France, UMR 8520 - IEMN - Institut \\ d'Electronique de Microélectronique et de Nanotechnologie, F-59000 Lille, France \\ ${ }^{2}$ Technical University of Ostrava, Nanotechnology Centre and IT4Innovations, Ostrava - Poruba, Czech Republic \\ Corresponding author: nicolas.tiercelin@iemn.fr
}

Polarization control of $\mathrm{THz}$ light is of paramount interest for the numerous applications offered in this frequency range. Recent developments in $\mathrm{THz}$ spintronic emitters allow for a very efficient broadband emission, and especially unique is their ability of $\mathrm{THz}$ polarization switching through magnetization control of the ferromagnetic layer[1]. So far, such a control has only been achieved using an external magnetic field. For the first time, we present here a scheme to achieve a voltage controlled coherent polarization rotation using a strain mediated magnetoelectric effect in spintronic emitters.

The considered emitter is a $\mathrm{W}(2 \mathrm{~nm}) / \mathrm{CoFe}(0.5 \mathrm{~nm}) / \mathrm{TbCo}_{2}(0.8 \mathrm{~nm}) / \mathrm{CoFe}(0.5 \mathrm{~nm}) / \mathrm{Pt}(2 \mathrm{~nm})$ stack deposited by RF sputtering in a LEYBOLD Z550 equipment on a $300 \mu \mathrm{m}$ thick $<011>$ cut PMN-PT ferroelectric relaxor. During the growth, a magnetic field was applied in the plane of the substrate in order to induce a well-defined uni-axial anisotropy that allows for a stoner-wohlfarth coherent rotation of the magnetization in the ferromagnetic layer. The $\mathrm{CoFe} / \mathrm{TbCo}_{2} / \mathrm{CoFe}$ tri-layer acts as an exchange-coupled multilayer and the $5 \mathrm{~d}$ metals Pt and $\mathrm{W}$ provide the ISHE with opposite signs for their spin-Hall angles.

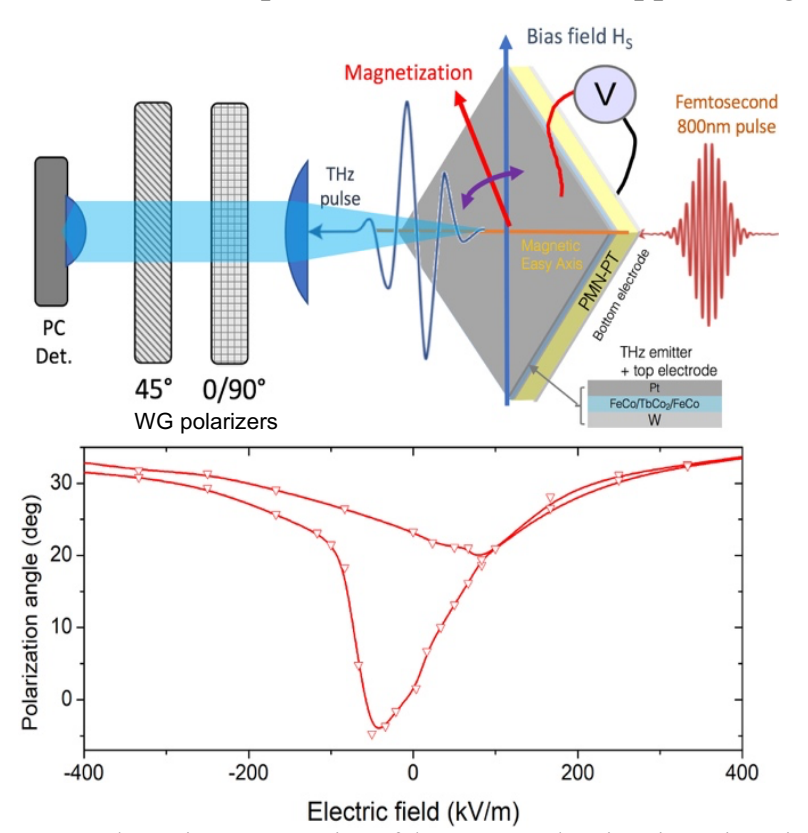

Fig. 1. Top: schematic representation of the magnetoelectric spintronic emitter and characterization setup. Bottom: measured E-polarization angle with respect to the horizontal plane, as a function of the applied voltage on PMN-PT.

The $\mathrm{THz}$ emission was characterized on a customized existing terahertz time-domain spectroscopy (TDS) setup. The ISHE-mediated terahertz emission is generated by pumping the sample with femtosecond pulses from a Ti:sapphire laser oscillator $(80 \mathrm{MHz}$ repetition rate, center wavelength $800 \mathrm{~nm}$ and $100 \mathrm{fs}$ pulse duration). The E-field of the emitted terahertz pulse is measured by sampling the response of a photo-conductive Auston switch that is probed by a split-off fraction of the femtosecond infrared pulse by a delay line. In order to measure the horizontal and vertical components of the E-field and deduce the polarization angle, two wire-grid polarizers were inserted in the $\mathrm{THz}$ path, as seen on figure 1 . Setting the delay line to obtain the maximum signal amplitude, Efield components were measured while cycling the applied voltage on the PMN-PT substrate. A static magnetic bias is also applied to the emitter. The deducted polarization angle is shown on figure 1, which clearly shows its controlled rotation over a span of nearly 40 degrees. Thanks to the magnetostrictive properties of the ferromagnetic tri-layer, and upon voltage application, the substrate generated stress induces a large change in magnetic anisotropy [2]. This in turn causes a coherent rotation of magnetization and thus of the emitted polarization. The hysteresis behavior reflects the hysteresis of the ferroelectric relaxor response.

\section{References:}

[1] Seifert, T. et al. Nature Photonics, 10(7), 483-488 (2016) https://doi.org/10.1038/nphoton.2016.91

[2] N. Tiercelin et al. Appl. Phys. Lett., 99, 192507 (2011) - doi: 10.1063/1.3660259 